\title{
An open-label pilot study of the effectiveness of adalimumab in patients with rheumatoid arthritis and previous infliximab treatment: relationship to reasons for failure and anti-infliximab antibody status
}

\author{
Arie E. van der Bijl • Ferdinand C. Breedveld • \\ Christian E. Antoni • Joachim R. Kalden • Sonja Kary • \\ Gerd R. Burmester • Christina Beckmann • \\ Kristina Unnebrink • Hartmut Kupper
}

Received: 28 November 2007 / Revised: 11 February 2008 / Accepted: 12 February 2008 /Published online: 19 March 2008

(C) The Author(s) 2008

\begin{abstract}
This prospective open-label pilot study evaluated the effectiveness and safety of adalimumab and the relationship to antibodies against infliximab (IFX) in adult patients with active rheumatoid arthritis (RA) who had been treated previously with IFX and experienced treatment failure owing to lack or loss of response or intolerance. Patients self-administered adalimumab $40 \mathrm{mg}$ subcutaneously every other week for 16 weeks, followed by maintenance therapy for up to Week 56. Measures of effectiveness included American College of Rheumatology (ACR) and European League Against Rheumatism (EULAR) response criteria, 28-joint Disease Activity Score, and the Health Assessment Questionnaire Disability Index. Serum IFX concentrations, human antichimeric antibody against IFX (HACA), adalimumab serum concentrations, antiadalimumab antibody, and safety also were
\end{abstract}

\footnotetext{
A. E. van der Bijl $(\bowtie) \cdot$ F. C. Breedveld

Department of Rheumatology, Leiden University Medical Center,

PO Box 9600, 2300 RC Leiden, The Netherlands

e-mail: a.e.van_der_Bijl@lumc.nl

C. E. Antoni · J. R. Kalden

University of Erlangen-Nuremberg,

Erlangen, Germany

S. Kary $\cdot$ G. R. Burmester

Charité University Medicine,

Berlin, Germany

C. Beckmann $\cdot$ K. Unnebrink $\cdot$ H. Kupper

Abbott GmbH \& Co. KG.,

Ludwigshafen, Germany
}

assessed. Of the 41 enrolled patients, 37 completed 16 weeks and 30 completed 56 weeks of treatment. Patients experienced clinically meaningful improvements in all measures of RA activity, with greater response rates observed for patients who had experienced loss of initial response to or intolerance of IFX. At Week 16, 46\% of patients achieved an ACR20 and 28\% achieved an ACR50; $61 \%$ achieved an at least moderate and $17 \%$ achieved a good EULAR response. Clinical benefit was maintained through Week 56 in all effectiveness parameters. Baseline HACA status did not significantly impact effectiveness. No new safety signals were observed; neither former IFX intolerance status nor baseline HACA status had a clinically relevant impact on adverse event frequency or severity. Adalimumab was effective and well-tolerated in patients with RA who previously failed IFX therapy, irrespective of reason for discontinuation and of HACA status.

Keywords Adalimumab - Infliximab .

Rheumatoid arthritis · Treatment failure .

Tumor necrosis factor antagonist

\section{Introduction}

Although tumor necrosis factor (TNF) antagonists are highly effective agents for the treatment of rheumatoid arthritis (RA), a subset of patients with RA may be intolerant to one of these agents or may experience an inadequate response or a loss of response over time [1-8]. A relevant clinical question, particularly as non-anti-TNF 
biologics have become available for RA therapy, is whether switching to a different TNF antagonist would be effective when the first has failed or resulted in intolerance [9]. Clinical reports to date demonstrated that a switch from one TNF antagonist to another is safe and effective [10-23]. Failure in effectiveness is recommended to be specified as an initial lack of response or loss of an initial response over time [16]. The formation of antibodies against infliximab (IFX) has been found to reduce the effectiveness of IFX over time [24].

This pilot study investigated the effectiveness and safety of adalimumab in patients with RA who had been treated previously with IFX and experienced treatment failure owing to no response, loss of response, or intolerance. The impact of anti-IFX antibodies on the subsequent therapy with adalimumab was also examined.

\section{Materials and methods}

\section{Patients}

This prospective open-label study included men and women $\geq 18$ years of age who met the American College of Rheumatology (ACR) criteria for the diagnosis of RA for at least 6 months, had active RA as defined by the 28-joint Disease Activity Score (DAS28) $\geq 3.2$ at study entry, and had previous use of IFX defined as administration of at least four infusions. The reasons for discontinuation of IFX were collected as no response, loss of response, or intolerance (i.e., infusion reactions) based on the investigator's assessment. Patients who discontinued IFX owing to adverse reactions other than intolerance were not allowed to enter the study. Additional exclusion criteria encompassed prior treatment with alkylating agents, intravenous immunoglobulin, or any investigational agent within 30 days or five half-lives (whichever was longer); IFX within the last 8 weeks; more than one disease-modifying antirheumatic drug (DMARD) or DMARD combination after IFX treatment; biologic RA therapies other than IFX; total lymphoid irradiation; history of malignancies; inflammatory joint disease other than RA; uncontrolled medical conditions (e.g., diabetes and congestive heart failure); active tuberculosis (TB), histoplasmosis, listeriosis, positive serology for hepatitis B or C, or human immunodeficiency virus; persistent infection or severe infection requiring hospitalization or recent treatment with intravenous or oral antibiotics; previous diagnosis or signs of central nervous demyelinating diseases; pregnancy; or breastfeeding.

If patients had chest radiographs indicative of prior TB infection or a positive purified protein derivative (PPD) tuberculin skin test, appropriate prophylactic anti-TB therapy had to be initiated before entering the study.
The protocol was approved by an independent ethics committee at participating sites, and written informed consent was obtained from all patients.

\section{Study design}

This was a 16-week open-label study that was followed by maintenance therapy for up to 56 weeks. After a screening period of at least 3 days (to allow for assessment of the PPD tuberculin skin test administered at the study entry visit), all patients self-administered adalimumab $40 \mathrm{mg}$ subcutaneously every other week (eow) in addition to their pre-existing antirheumatic therapy. Doses of DMARDs, glucocorticoids (prednisone equivalent $\leq 10 \mathrm{mg} /$ day), and/or nonsteroidal anti-inflammatory drugs that patients may have been taking at study entry were to remain stable until Week 16. Patients were to be withdrawn from the study if they did not show adequate response to study treatment at Week 16 (less than 20\% reduction in tender joint count [TJC] and/or swollen joint count [SJC] compared with study entry). Addition of further DMARDs was not allowed.

Effectiveness, pharmacokinetic, and safety evaluations

Patients were assessed at screening, and then at baseline, Weeks 2,8 , and 16 , and every 8 weeks thereafter through Week 56. Effectiveness assessments included the change in DAS28 compared with study entry and both European League Against Rheumatism (EULAR) and ACR response criteria, the Health Assessment Questionnaire Disability Index (HAQ DI), and acute-phase reactants. The effectiveness results of Week 16 were considered primary.

Adverse events (AEs) were collected at each visit after the first adalimumab injection up to 70 days after the last injection.

Serum concentrations of adalimumab were analyzed at baseline and at Weeks 16, 40, and 56 using a validated enzyme-linked immunosorbent assay (ELISA) method by Abbott Laboratories. The lower limit of quantitation (LLOQ) for adalimumab was established at $31.3 \mathrm{ng} / \mathrm{ml}$ in undiluted serum. Antiadalimumab antibody (AAA) was measured at study entry and at Weeks 16,40 , and 56 using a validated double-antigen ELISA. The LLOQ for AAA was established at $5 \mathrm{ng} / \mathrm{ml}$ in undiluted serum. A positive antiadalimumab signal was defined as a concentration exceeding $20 \mathrm{ng} / \mathrm{ml}$ that could not be suppressed by $\geq 50 \%$ after the addition of $10 \%$ human serum to the sample. Human antichimeric antibody (HACA; i.e., antiIFX antibodies) and serum IFX concentrations status were determined at baseline using ELISA methods. Because the presence of adalimumab interferes with the serum IFX ELISA assay, HACA and serum IFX concentrations were not assessed beyond baseline. 
Statistical analysis

The sample size of this open-label pilot study was based on practical rather than biometric considerations. Both the effectiveness and safety analyses were performed on the set of patients who received at least one injection of adalimumab. Subgroup analyses for effectiveness endpoints also were performed by reason for discontinuation of IFX and by HACA status at baseline. In the case of discontinuation of IFX owing to no response or loss of response, patients were counted in the corresponding group regardless of whether they had an additional cause of intolerance, whereas the IFX discontinuation owing to intolerance subgroup was exclusive.

Effectiveness analysis consisted of summary statistics and confidence intervals with last-observation-carried-forward methods applied. Pharmacokinetic analysis consisted of descriptive statistics.

\section{Results}

Patient disposition and baseline characteristics

Of 41 patients enrolled in the study, 37 (90.2\%) completed 16 weeks of treatment. A total of 30 patients $(73.2 \%)$ were still receiving adalimumab at the Week-56 visit. Of the 11 patients who withdrew from the study before Week 56 , six $(14.6 \%)$ withdrew owing to the occurrence of one or more AEs and seven (17.1\%) withdrew owing to a lack of effectiveness (more than one reason for discontinuation was possible).

The majority of patients were white $(95 \%)$ and female $(88 \%)$, with a mean age of 55 years. The mean duration of RA for all treated patients was 12 years, and the mean/ median duration of prior IFX treatment was 17/13 months. The mean (standard deviation) period between the last IFX infusion and first adalimumab administration was $13 \pm$ 5.3 weeks, with a median of 11 weeks. A total of 19 patients had received their last IFX infusion more than 12 weeks before the first adalimumab injection. Table 1 summarizes the demographic and RA disease characteristics for the study population, by reason of discontinuation of IFX and by HACA status.

A total of 27 (66\%) patients were receiving DMARDs at baseline, with methotrexate being the most common ( 25 patients). Measurable serum HACA concentrations were found in 17 of 41 (41.5\%) patients at baseline. Nine patients were not assessable for HACA status owing to remaining IFX serum concentrations. Each of these nine patients had discontinued therapy because of unsatisfactory response (either no response or loss of response). The pattern of reasons for discontinuation of IFX was somewhat variable, with six $(40 \%)$ of 15 patients who were HACA negative experiencing no response to IFX compared with four $(23.5 \%)$ of 17 patients who were HACA positive experiencing no response to IFX (Table 2).

A total of six (14.6\%) patients received isoniazid prophylaxis for TB based on baseline chest radiograph indicative of latent TB, positive PPD skin test, or other risk factors.

Table 1 Disease severity characteristics and prior infliximab/DMARD administration by reason for discontinuation of prior infliximab and HACA status

\begin{tabular}{|c|c|c|c|c|c|c|}
\hline \multirow[t]{2}{*}{ Parameter $^{\mathrm{a}}$} & \multicolumn{4}{|c|}{ Reason for discontinuation of prior infliximab } & \multicolumn{2}{|c|}{ HACA status ${ }^{b}$} \\
\hline & $\begin{array}{l}\text { All patients } \\
(N=41)\end{array}$ & $\begin{array}{l}\text { No response } \\
(n=15)\end{array}$ & $\begin{array}{l}\text { Loss of response } \\
(n=21)\end{array}$ & $\begin{array}{l}\text { Intolerance } \\
(n=5)\end{array}$ & $\begin{array}{l}\text { Positive } \\
(N=17)\end{array}$ & $\begin{array}{l}\text { Negative } \\
(N=15)\end{array}$ \\
\hline Mean duration of RA, $y$ & $11.6 \pm 7.4$ & $12.2 \pm 9.2$ & $11.8 \pm 7.0$ & $9.2 \pm 2.5$ & $9.6 \pm 4.5$ & $15.4 \pm 9.1$ \\
\hline Current use of 1 DMARD, $n(\%)$ & $27(66)$ & $10(67)$ & $14(67)$ & $3(60)$ & $9(53)$ & $11(73)$ \\
\hline Mean duration of infliximab treatment, mo & $17.3 \pm 15.1$ & $9.3 \pm 5.3$ & $23.4 \pm 17.6$ & $15.6 \pm 14.0$ & $12.8 \pm 8.8$ & $20.2 \pm 18.6$ \\
\hline Mean dose per infliximab infusion, mg & $262.4 \pm 87.6$ & $263.7 \pm 81.9$ & $267.5 \pm 100.0$ & $237.2 \pm 47.8$ & $279.6 \pm 81.5$ & $264.3 \pm 111.2$ \\
\hline Mean interval last infliximab/first adalimumab, week & $13.0 \pm 5.3$ & $13.4 \pm 5.2$ & $11.9 \pm 5.1$ & $16.4 \pm 5.8$ & $14.9 \pm 6.2$ & $12.0 \pm 4.8$ \\
\hline DAS28 & $6.1 \pm 0.9$ & $5.9 \pm 0.7$ & $6.2 \pm 1.0$ & $6.5 \pm 0.9$ & $6.3 \pm 0.9$ & $6.1 \pm 0.9$ \\
\hline Tender joint count ( 28 joints) & $14.8 \pm 6.6$ & $13.7 \pm 6.9$ & $14.7 \pm 6.6$ & $18.6 \pm 4.7$ & $14.6 \pm 6.6$ & $14.6 \pm 7.2$ \\
\hline Swollen joint count ( 28 joints) & $8.2 \pm 4.8$ & $5.9 \pm 4.1$ & $9.0 \pm 4.6$ & $12.0 \pm 4.4$ & $9.7 \pm 4.4$ & $8.5 \pm 5.5$ \\
\hline HAQ DI & $1.85 \pm 0.49$ & $1.92 \pm 0.52$ & $1.80 \pm 0.43$ & $1.85 \pm 0.71$ & $1.89 \pm 0.50$ & $1.79 \pm 0.48$ \\
\hline $\mathrm{CRP}, \mathrm{mg} / \mathrm{l}$ & $25.1 \pm 32.0$ & $23.3 \pm 26.8$ & $26.3 \pm 38.1$ & $26.0 \pm 21.4$ & $29.2 \pm 28.8$ & $29.3 \pm 40.5$ \\
\hline
\end{tabular}

CRP C-reactive protein; DAS28 28-joint Disease Activity Score; DMARD disease-modifying antirheumatic drug; HACA human antichimeric (anti-infliximab) antibody; HAQ DI Health Assessment Questionnaire Disability Index; RA rheumatoid arthritis

${ }^{\text {a }}$ Mean \pm SD except where otherwise specified.

${ }^{\mathrm{b}}$ Not assessable in nine patients because of the presence of infliximab serum concentrations. 
Table 2 Baseline HACA status and infliximab serum concentration status by reason for discontinuation of prior infliximab

\begin{tabular}{|c|c|c|c|c|}
\hline \multirow[t]{2}{*}{ Reason for discontinuation of prior infliximab } & \multicolumn{2}{|l|}{ HACA status ${ }^{\mathrm{a}}$} & \multicolumn{2}{|c|}{ Measurable infliximab serum concentration at study entry } \\
\hline & Positive $(n=17)$ & Negative $(n=15)$ & Yes $(n=9)$ & No $(n=32)$ \\
\hline No response & 4 & 6 & 4 & 10 \\
\hline Loss of response & 10 & 7 & 5 & 17 \\
\hline Intolerance & 3 & 2 & 0 & 5 \\
\hline
\end{tabular}

HACA human antichimeric (anti-infliximab) antibody

${ }^{a}$ In nine patients, HACA could not be determined because of measurable infliximab serum concentrations (see second column from right).

\section{Effectiveness}

\section{All patients}

Clinically meaningful improvements occurred in all measures of RA activity in the overall patient popula- tion after 16 weeks of adalimumab treatment (Table 3). The ACR and EULAR response rates were maintained or increased through 56 weeks (Fig. 1). Similarly, the DAS28, the HAQ DI, TJC, SJC, and C-reactive protein (CRP) concentrations improved from baseline to Week 16 (Table 3).

Table 3 Adalimumab efficacy at week 16 (LOCF data)

\begin{tabular}{|c|c|c|c|c|c|c|}
\hline & \multirow{2}{*}{$\begin{array}{l}\text { All patients }{ }^{\mathrm{a}} \\
(N=41)\end{array}$} & \multicolumn{3}{|c|}{ Reason for discontinuation of prior infliximab ${ }^{a}$} & \multicolumn{2}{|l|}{ HACA status ${ }^{\mathrm{c}}$} \\
\hline & & $\begin{array}{l}\text { No response } \\
(n=15)\end{array}$ & $\begin{array}{l}\text { Loss of response } \\
(n=21)\end{array}$ & $\begin{array}{l}\text { Intolerance }^{\mathrm{b}} \\
(n=5)\end{array}$ & $\begin{array}{l}\text { Positive } \\
(n=17)\end{array}$ & $\begin{array}{l}\text { Negative } \\
(n=15)\end{array}$ \\
\hline ACR20 response, $\%$ & 46 & 29 & 60 & 40 & 47 & 43 \\
\hline ACR50 response, $\%$ & 28 & 14 & 40 & 20 & 47 & 7 \\
\hline ACR70 response, $\%$ & 13 & 7 & 20 & 0 & 18 & 7 \\
\hline Moderate EULAR response, $\%$ & 61 & 47 & 67 & 80 & 53 & 53 \\
\hline Good EULAR response, $\%$ & 17 & 7 & 24 & 20 & 24 & 7 \\
\hline \multicolumn{7}{|c|}{ DAS28, mean change from baseline } \\
\hline Absolute change $\pm \mathrm{SD}$ & $-1.5 \pm 1.6$ & $-1.0 \pm 0.9$ & $-1.8 \pm 2.0$ & $-1.4 \pm 0.6$ & $-1.6 \pm 1.8$ & $-1.1 \pm 1.6$ \\
\hline Percent change & -23 & -16 & -28 & -23 & -25 & -17 \\
\hline DAS28 mean value & 4.6 & 4. 9 & 4.4 & 5.0 & 4.7 & \\
\hline \multicolumn{7}{|l|}{$\begin{array}{l}\text { HAQ DI score }(0-3), \\
\text { mean change from baseline }\end{array}$} \\
\hline Absolute change $\pm \mathrm{SD}$ & $-0.21 \pm 0.50$ & $-0.13 \pm 0.53$ & $-0.36 \pm 0.48$ & $-0.15 \pm 0.34$ & $-0.36 \pm 0.52$ & $-0.08 \pm 0.43$ \\
\hline Percent change & -11 & -7.5 & -20 & -5 & -18 & -3 \\
\hline HAQ DI mean value & 1.64 & 1.79 & 1.42 & 1.70 & 1.53 & 1.88 \\
\hline \multicolumn{7}{|l|}{$\begin{array}{l}\text { Tender joint count ( } 0-28 \text { joints), } \\
\text { mean change from baseline }\end{array}$} \\
\hline Absolute change $\pm \mathrm{SD}$ & $-6.8 \pm 8.3$ & $-5.1 \pm 4.6$ & $-7.2 \pm 10.6$ & $-10.0 \pm 5.7$ & $-7.2 \pm 9.3$ & $-5.6 \pm 8.8$ \\
\hline Percent change & -38 & -36 & -37 & -54 & -40 & -30 \\
\hline \multicolumn{7}{|l|}{$\begin{array}{l}\text { Swollen joint count ( } 0-28 \text { joints), } \\
\text { mean change from baseline }\end{array}$} \\
\hline Absolute change $\pm \mathrm{SD}$ & $-4.6 \pm 5.1$ & $-2.9 \pm 2.7$ & $-6.1 \pm 5.0$ & $-7.8 \pm 4.7$ & $-5.5 \pm 4.8$ & $-4.2 \pm 6.6$ \\
\hline Percent change & -53 & -47 & -65 & -60 & -48 & -48 \\
\hline \multicolumn{7}{|c|}{ CRP (mg/l), mean change from baseline } \\
\hline Absolute change $\pm \mathrm{SD}$ & $-3.8 \pm 19.5$ & $-5.4 \pm 18.8$ & $-1.1 \pm 21.3$ & $-10.6 \pm 13.3$ & $-6.3 \pm 22.4$ & $-0.8 \pm 18.7$ \\
\hline Percent change & -21 & -15 & -40 & -40 & -17 & -34 \\
\hline
\end{tabular}

$A C R$ American College of Rheumatology; DAS28 28-joint Disease Activity Score; EULAR European League Against Rheumatism; HACA human antichimeric antibody (anti-infliximab); HAQ DI Health Assessment Questionnaire Disability Index; CRP C-reactive protein (reference 4 mg/l) ${ }^{a}$ Total number of patients enrolled; last observation carried forward (LOCF).

${ }^{\mathrm{b}}$ Those patients who had discontinued prior infliximab treatment for both an efficacy and safety reason were assigned to the corresponding efficacy subgroup. The intolerance subgroup consists of patients who had discontinued prior infliximab treatment strictly because of intolerance. ${ }^{\mathrm{c}}$ Nine patients were not assessable for HACA owing to measurable infliximab concentrations. 


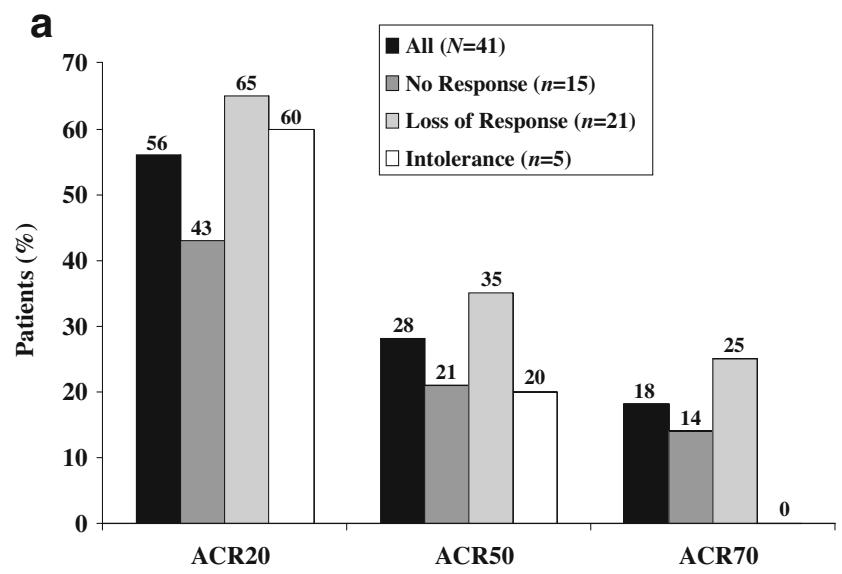

b

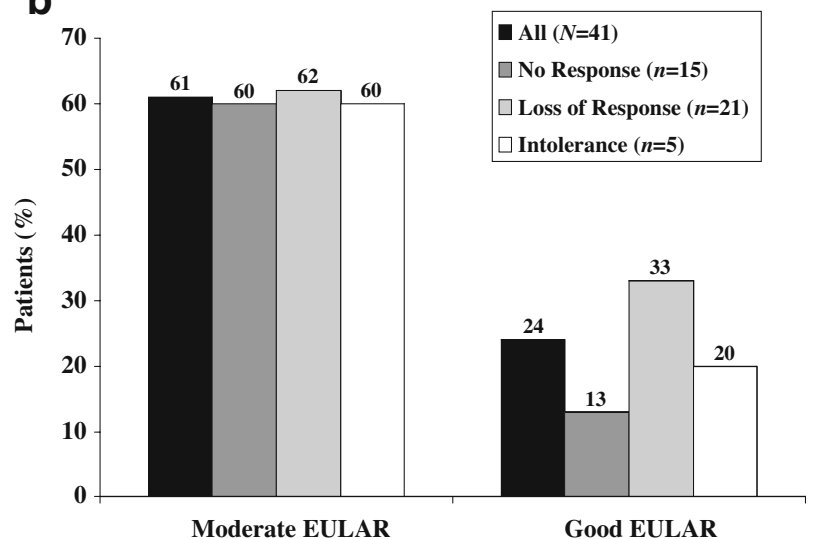

Fig. 1 a American College of Rheumatology 20\% (ACR20), ACR50, and ACR70 responses and b European League Against Rheumatism $(E U L A R)$ responses with adalimumab treatment at Week 56 (last observation carried forward) by reason for discontinuation of prior infliximab

\section{Effectiveness by reason for discontinuation of prior IFX therapy}

Adalimumab treatment led to clinically relevant improvement in disease activity irrespective of the reason for stopping prior IFX therapy. However, the intolerance group was small, which limits the relevance of the results. At Week 16, ACR20 was $29 \%, 60 \%$, and $40 \%$ among those patients who had no response, loss of response, and intolerance to IFX, respectively (Table 3). By Week 56, an increase in response rates was found across all subsets (Fig. 1a). At Week 16, a moderate EULAR response was achieved by $47 \%, 67 \%$, and $80 \%$ of patients who had no response, a loss of response, and intolerance to IFX, respectively (Table 3 ). The rates of moderate EULAR response increased in all subsets through Week 56. A good EULAR response was achieved at Week 16 by $7 \%, 24 \%$, and $20 \%$ of patients with no response, loss of response, and intolerance, respectively. The rates of patients with good EULAR response increased up to Week 56 (Fig. 1b). The DAS28 and the HAQ DI improved from baseline in all three subgroups at all time points evaluated, with the greatest response occurring in those patients who had stopped IFX owing to loss of response (Week 16 data shown in Table 3 ).

All three subgroups experienced improvements from baseline in both TJC and SJC at all time points measured, with those who reported intolerance to IFX or loss of response achieving a slightly greater benefit compared with those who had no response to IFX (Table 3). A similar pattern of relative change was found for the reduction of CRP concentrations (Table 3).

\section{Effectiveness by HACA status}

At 16 weeks, a total of $47 \%$ of HACA-positive patients and $43 \%$ of HACA-negative patients achieved an ACR20 response, whereas $47 \%$ and $7 \%$ of these patient subgroups, respectively, achieved an ACR50 response (Table 3). Similarly, $53 \%$ of HACA-positive patients and $53 \%$ of HACA-negative patients achieved a moderate EULAR response at 16 weeks (Table 3). Both HACA-positive and HACA-negative patients achieved clinically important mean changes in other measures of disease activity, including TJC and SJC, DAS28, and HAQ DI (Table 3). The response rates markedly increased continuously from Week 24 (not shown) up to Week 56 in patients with negative HACA status at baseline and were sustained in patients with positive HACA status (Fig. 2).

\section{Pharmacokinetics}

From Weeks 17 to 45 of the study, eight of 41 patients (19.5\%) switched to $40 \mathrm{mg}$ weekly administration of adalimumab based on disease severity and investigator's

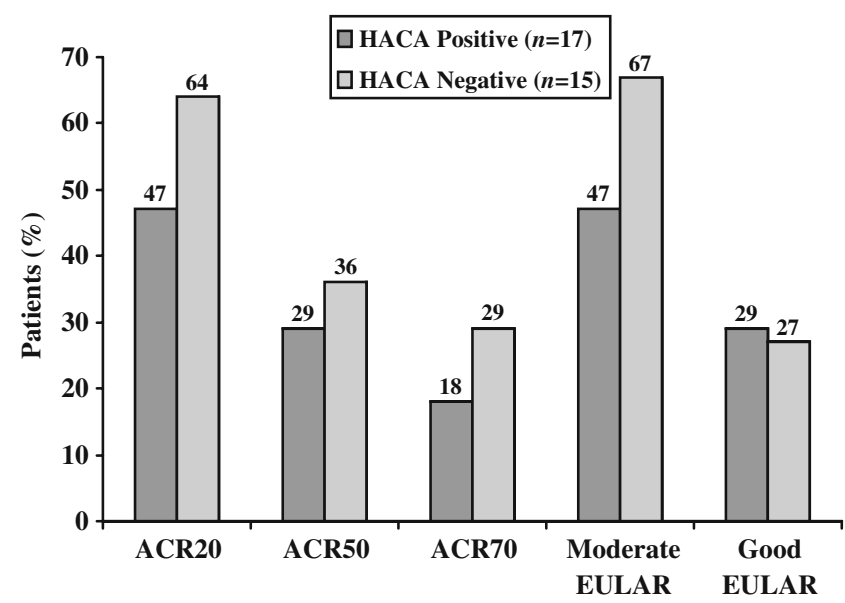

Fig. 2 American College of Rheumatology 20\% (ACR20), ACR50, ACR70 response and European League Against Rheumatism (EULAR) response rates to adalimumab treatment at Week 56 (last observation carried forward) by human antichimeric (anti-infliximab) antibody $(H A C A)$ status at baseline 
request. Twelve of 14 (86\%) patients receiving adalimumab $40 \mathrm{mg}$ eow without concomitant DMARD and 21 of 25 patients (84\%) receiving adalimumab $40 \mathrm{mg}$ eow with methotrexate remained on the original dose regimen for the duration of the study. The summary statistics of serum adalimumab concentrations for patients remaining on the original dosage are provided in Table 4. Mean serum trough adalimumab concentrations in these patients were within or slightly above the 4- to $8-\mu \mathrm{g} / \mathrm{ml}$ range, which is the typical trough concentration for the recommended regimen of adalimumab $40 \mathrm{mg}$ eow [25]. For patients with measurable HACA at baseline, mean serum adalimumab trough concentrations ranged from 1 to $4 \mu \mathrm{g} / \mathrm{ml}$, which is lower than those achieved in patients without measurable HACA levels and below the typical trough concentration for $40 \mathrm{mg}$ eow (data not shown). As noted earlier (see "Effectiveness by HACA status"), baseline HACA status did not appear to have a major impact on effectiveness outcome parameters. Two patients (4.9\%, both of whom had measurable HACA concentrations at baseline) developed AAA and both responded well to adalimumab (both without concomitant DMARD treatment), with a DAS28 change from baseline to Week 56 of -1.5 in one patient (AAA present at Week 16 only, despite weekly adalimumab injections beyond Week 16) and of -5.4 in the other patient (AAA present at Weeks 40 and 56, with no dose increase of adalimumab).

\section{Safety}

Overall, 43 events were reported by 34 patients during 47 weeks of mean exposure to adalimumab (37 patientyears), the most frequent being RA or nasopharyngitis. Neither former IFX intolerance status nor baseline HACA status appeared to have a clinically meaningful impact on the frequency or severity of allergic reactions or other AEs.
No demyelinating disorders or other autoimmune disorders, including lupus-like reactions, were reported. One patient with a history of hypertension died during the study as a result of intracerebral bleeding. This event was considered to be probably unrelated to study drug by the investigator. One patient developed pulmonary TB during the study period. Approximately 6 months after study entry, the patient developed dry cough; 5 additional months later, TB was diagnosed based on a positive polymerase chain reaction of the sputum. At screening, the patient, who had contact with a person with active TB 10 years before, had no abnormalities in the chest X-ray and a negative PPD test result. Although night sweats and cough had occurred during the former IFX treatment as well, TB was not diagnosed at that time. The event resolved after 9 months of anti-TB therapy. One other serious infection (cellulitis) was reported. Two patients reported the development of lymphomas (one large B cell lymphoma after three injections of adalimumab, one $\mathrm{T}$ cell lymphoma after 1 year of adalimumab treatment in a patient with RA and Sjögren's disease) during the study. Both events were considered either unrelated or probably unrelated to study drug administration by the investigator owing to the short exposure to adalimumab, the history of previous exposure to other immunosuppressive agents, and the underlying RA. Coincidentally, one of the two lymphoma cases occurred in the same patient who was diagnosed with TB.

\section{Discussion}

In accordance with other reports about successful switching from one TNF antagonist to another, the results of this study indicate that patients with RA who had previously discontinued treatment with IFX experienced clinically meaningful improve-

Table 4 Summary statistics of serum adalimumab concentrations $(\mu \mathrm{g} / \mathrm{ml})$ for patients remaining on the original dose regimen $(40 \mathrm{mg}$ every other week; $N=33$ )

\begin{tabular}{|c|c|c|c|c|c|c|}
\hline & \multicolumn{6}{|l|}{ Week } \\
\hline & \multicolumn{3}{|c|}{ Without methotrexate } & \multicolumn{3}{|c|}{ With methotrexate } \\
\hline & Baseline $^{\mathrm{a}}$ & Week 16 & Week 56 & Baseline $^{\mathrm{a}}$ & Week 16 & Week 56 \\
\hline$N$ & 12 & 10 & 5 & 21 & 19 & 17 \\
\hline Mean & - & 4.3 & 7.2 & - & 6.3 & 7.1 \\
\hline SD & - & 4.0 & 5.9 & - & 3.6 & 3.9 \\
\hline Minimum & - & 0.0 & 0.0 & - & 0.4 & 0.0 \\
\hline Median & - & 3.1 & 5.0 & - & 5.8 & 7.6 \\
\hline Maximum & - & 10.6 & 13.6 & - & 14.6 & 12.6 \\
\hline $\mathrm{CV} \%$ & - & 93.6 & 81.1 & - & 56.7 & 54.4 \\
\hline
\end{tabular}

$C V$ Coefficient of variation

${ }^{\text {a }}$ For nine patients who had measurable infliximab results at baseline, baseline adalimumab concentrations were not included in the summary statistics because there is potential for infliximab to interfere with the adalimumab assay. 
ments in all effectiveness endpoints with adalimumab treatment [10-15, 17-19]. The patients who reported a loss of response under IFX treatment appeared to experience the greatest effectiveness with adalimumab by several disease-response measures, followed by the small patient subset that had been intolerant to IFX. The patients with no response to their previous IFX therapy had somewhat lower response rates to adalimumab. Similar results were recently published in another open-label study in a large patient cohort who switched from prior anti-TNF therapy to adalimumab [18]. The documentation of reason for discontinuation of IFX is limited, as there was no other criterion but the investigator's clinical assessment.

Interestingly, patients with a reported initial lack of response to IFX had the lowest mean DAS28 value and lowest joint counts at baseline among the subsets by reason of IFX discontinuation, whereas the mean HAQ DI at baseline was worst in these patients. However, most of the recent publications either from registers or from clinical studies on switching among TNF antagonists are based on the physician's discretion when the reasons for discontinuation are reported [17-19].

Results of subgroup analyses by patient's baseline HACA status (available for 32 of the 41 patients) indicate that adalimumab is effective and well-tolerated in patients who may have developed HACA with prior IFX treatment. The low ACR50 and ACR70 and good EULAR response rates at Week 16 in patients with negative HACA appear to be more likely related to confounding baseline characteristics, such as a greater percentage of patients who had experienced no response to IFX and a longer RA duration compared with patients with positive HACA status. However, beyond Week 16, the response rates in the HACA-negative subset increased and even surpassed the rates achieved by HACA-positive patients.

Results of the pharmacokinetic analysis indicate that, for the majority of patients, the mean steady-state serum adalimumab trough concentrations achieved with the recommended regimen of $40 \mathrm{mg}$ eow were near or above 4 to $8 \mu \mathrm{g} / \mathrm{ml}$, which is consistent with what has been observed in other pharmacokinetic trials of adalimumab in patients with RA $[26,27]$. These concentrations are three to seven times the average effective concentration in $50 \%$ of patients for TJC, SJC, and numeric ACR [27]. Although mean serum adalimumab trough concentrations appeared to be lower in patients with measurable HACA at baseline, the potential for interference of HACA with the serum adalimumab assay has not been evaluated. In general, the mean and median serum adalimumab concentrations were greater in patients who concomitantly received methotrexate. In a recent study in 121 patients with RA treated with adalimumab, good responders had greater adalimumab serum concentrations than nonresponders, and the forma- tion of AAA (present in 21/121) was reduced in patients who received concomitant methotrexate [28]. AAAs were detected in two of the patients in this analysis, both were not receiving concomitant DMARDs and both had a positive HACA status at study entry. In previous clinical adalimumab trials, AAA had been documented in $12 \%$ of the patients and was not found to have an impact on the efficacy of adalimumab [29].

Adalimumab was generally well-tolerated in this selected population, and safety results did not suggest any new signals with regard to the safety of the drug. In particular, no additional risks for allergic reactions under adalimumab were found in these 41 patients with a history of intolerance to IFX.

In the two patients diagnosed with lymphomas (cutaneous $\mathrm{T}$ cell lymphoma in one and a B cell lymphoma in the other), exposure to adalimumab was $\leq 1$ year. The total exposure to anti-TNF therapy and the previous extensive immunosuppressive treatment for RA together in these patients represent a risk for the development of lymphoma.

Limitations of our study include the small number of patients in each subgroup and the nonrandomized study design.

The results of this pilot study indicate that adalimumab is generally effective and well-tolerated for the treatment of RA in patients who have failed IFX therapy, including those who have developed HACA for IFX.

Acknowledgments The authors thank the physicians and staff members of all study centers for having participated in this study. In addition, we thank Susan Paulson, PhD, for the performance of pharmacokinetic analyses, and Angela Cimmino, PharmD, who provided writing assistance on behalf of Abbott Laboratories. The study was sponsored by Abbott Laboratories.

Conflicts of interest statement Arie E. van der Bijl has no conflicts of interest pertaining to this article. Ferdinand Breedveld has received consulting fees or honoraria (less than $\$ 10,000$ per year) from Centocor, Schering-Plough, Amgen/Wyeth, and Abbott. Christian Antoni received research grants, consulting fees, and other remuneration from Centocor, Schering-Plough, and Abbott Laboratories. Employee of Scherring-Plough. Joachim Kalden is active as a consultant for Abbott, MSD, Roche, Wyeth, Centocor, and ScheringPlough. Sonja Kary is an employee and holds stock/stock options for Abbott Laboratories; Consulting fees - Bristol Meyer Squibb. Gerd Burmester has received honoraria and consulting fees from Abbott and is on the Abbott speakers bureau. Christina Beckmann is employed by Abbott Laboratories. Kristina Unnebrink is employed by Abbott Laboratories and holds Abbott stock options. Hartmut Kupper is employed by Abbott Laboratories and holds Abbott stock options.

Open Access This article is distributed under the terms of the Creative Commons Attribution Noncommercial License which permits any noncommercial use, distribution, and reproduction in any medium, provided the original author(s) and source are credited.

\section{References}

1. Breedveld FC, Weisman MH, Kavanaugh AF et al (2006) The PREMIER study: A multicenter, randomized, double-blind clinical 
trial of combination therapy with adalimumab plus methotrexate versus methotrexate alone or adalimumab alone in patients with early, aggressive rheumatoid arthritis who had not had previous methotrexate treatment. Arthritis Rheum 54:26-37

2. Genovese MC, Bathon JM, Fleischmann RM et al (2005) Longterm safety, efficacy, and radiographic outcome with etanercept treatment in patients with early rheumatoid arthritis. J Rheumatol 32:1232-1242

3. Keystone EC, Kavanaugh AF, Sharp JT et al (2004) Radiographic, clinical, and functional outcomes of treatment with adalimumab (a human anti-tumor necrosis factor monoclonal antibody) in patients with active rheumatoid arthritis receiving concomitant methotrexate therapy: a randomized, placebo-controlled, 52-week trial. Arthritis Rheum 50:1400-1411

4. Navarro-Sarabia F, Ariza-Ariza R, Hernandez-Cruz B et al (2005) Adalimumab for treating rheumatoid arthritis. Cochrane Database Syst Rev 3:CD005113

5. Smolen JS, van der Heijde DM, St Clair EW et al (2006) Predictors of joint damage in patients with early rheumatoid arthritis treated with high-dose methotrexate with or without concomitant infliximab: results from the ASPIRE trial. Arthritis Rheum 54:702-710

6. St. Clair EW, van der Heijde DM, Smolen JS et al (2004) Activecontrolled study of patients receiving infliximab for the treatment of rheumatoid arthritis of early onset study group. Combination of infliximab and methotrexate therapy for early rheumatoid arthritis: a randomized, controlled trial. Arthritis Rheum 50:3432-3443

7. van der Heijde D, Klareskog L, Rodriguez-Valverde V et al (2006) Comparison of etanercept and methotrexate, alone and combined, in the treatment of rheumatoid arthritis: two-year clinical and radiographic results from the TEMPO study, a double-blind, randomized trial. Arthritis Rheum 54:1063-1074

8. Nurmohamed MT, Dijkmans BA (2005) Efficacy, tolerability and cost effectiveness of disease-modifying antirheumatic drugs and biologic agents in rheumatoid arthritis. Drugs 65:661-694

9. Finckh A, Ciurea A, Brulhart L et al (2007) B cell depletion may be more effective than switching to an alternative anti-tumor necrosis factor agent in rheumatoid arthritis patients with inadequate response to anti-tumor necrosis factor agents. Arthritis Rheum 56:1417-1523

10. Brocq O, Albert C, Roux C et al (2004) Adalimumab in rheumatoid arthritis after failed infliximab and/or etanercept therapy: experience with 18 patients. Joint Bone Spine 71:601-603

11. Gomez-Reino JJ, Carmona L, for the BIOBADASER Group (2006) Switching TNF antagonists in patients with chronic arthritis: an observational study of 488 patients over a four-year period. Arthritis Res Ther 8:R29

12. Hansen KE, Hildebrand JP, Genovese MC et al (2004) The efficacy of switching from etanercept to infliximab in patients with rheumatoid arthritis. J Rheumatol 31:1098-1102

13. Haraoui B, Keystone EC, Thorne JC et al (2004) Clinical outcomes of patients with rheumatoid arthritis after switching from infliximab to etanercept. J Rheumatol 31:2356-2359

14. Nikas SN, Voulgari PV, Alamanos Y et al (2006) Efficacy and safety of switching from infliximab to adalimumab: a comparative controlled study. Ann Rheum Dis 65:257-260
15. van Vollenhoven R, Harju A, Brannemark S et al (2003) Treatment with infliximab (Remicade) when etanercept (Enbrel) has failed or vice versa: data from the STURE registry showing that switching tumour necrosis factor $\alpha$ blockers can make sense. Ann Rheum Dis 62:1195-1198

16. Van Vollenhoven RF (2004) Switching between biologic agents. Clin Exp Rheumatol 22(Suppl 35):S115-S121

17. Hyrich KL, Lunt M, Watson K et al (2007) Outcomes after switching from one anti-tumor necrosis factor $\alpha$ agent to a second anti-tumor necrosis factor $\alpha$ agent in patients with rheumatoid arthritis. Arthritis Rheum 56:13-20

18. Bombardieri S, Ruiz AA, Fardellone P, for the Research in Active Rheumatoid Arthritis (ReAct) Study Group et al (2007) Effectiveness of adalimumab for rheumatoid arthritis in patients with a history of TNF-antagonist therapy in clinical practice. Rheumatology (Oxford) 46:1191-1199

19. Hjardem E, Ostergaard M, Pødenphant J et al (2007) Do rheumatoid arthritis patients in clinical practice benefit from switching from infliximab to a second tumor necrosis factor alpha inhibitor? Ann Rheum Dis 66:1184-1189

20. Cohen G, Courvoisier N, Cohen JD et al (2005) The efficacy of switching from infliximab to etanercept and vice-versa in patients with rheumatoid arthritis. Clin Exp Rheumatol 23:795-800

21. Gomez-Puerta JA, Sanmarti R, Rodriguez-Cros JR et al (2004) Etanercept is effective in patients with rheumatoid arthritis with no response to infliximab therapy. Ann Rheum Dis 63:896

22. Wick MC, Ernestam S, Lindblad S et al (2005) Adalimumab (Humira) restores clinical response in patients with secondary loss of efficacy from infliximab (Remicade) or etanercept (Enbrel): results from the STURE registry at Karolinska University Hospital. Scand J Rheumatol 34:353-358

23. Bennett AN, Peterson P, Zain A et al (2005) Adalimumab in clinical practice. Outcome in 70 rheumatoid arthritis patients, including comparison of patients with and without previous antiTNF exposure. Rheumatology (Oxford) 44:1026-1031

24. Wolbrink GJ, Vis M, Lems WF et al (2006) Development of antiinfliximab antibodies and relationship to clinical response in patients with rheumatoid arthritis. Arthritis Rheum 54:711-715

25. Humira ${ }^{\circledR}$ [package insert] (2006) Chicago, IL: Abbott Laboratories

26. Awni WM, Cascella P, Oleka N et al (2003) Steady-state pharmacokinetics of adalimumab (Humira) following $40 \mathrm{mg}$ subcutaneous injection every other week in rheumatoid arthritis patients with and without methotrexate background therapy [poster 255]. Arthritis Rheum 48(Suppl):S140

27. Granneman RG, Zhang Y, Noertersheuser PA et al (2003) Pharmacokinetic/pharmacodynamic relationships of adalimumab (Humira) in rheumatoid arthritis patients during phase II/III clinical trials [poster 256]. Arthritis Rheum 48(Suppl):S140

28. Bartelds GM, Wijbrandts CA, Nurmohamed MT et al (2007) Clinical response to adalimumab: Relationship to anti-adalimumab antibodies and serum adalimumab concentrations in rheumatoid arthritis. Ann Rheum Dis 66:921-926

29. van de Putte LBA, Atkins C, Malaise M et al (2004) Efficacy and safety of adalimumab as monotherapy in patients with rheumatoid arthritis for whom previous disease modifying antirheumatic drug treatment has failed. Ann Rheum Dis 63:508-516 